\title{
The Limitations of Certain Density Functionals in Modeling Neutral Water Clusters
}

\author{
George C. Shields and Karl N. Kirschner \\ Department of Chemistry, Center for Molecular Design, Hamilton College, Clinton, New York, USA
}

\begin{abstract}
We have used density functional theory (DFT) with four different functionals and with basis sets optimized for studying water clusters to calculate the structures and energies for selected water dimer, trimer, tetramer, pentamer, hexamer, and octamer structures. We compare the results to the CBS-APNO and G3 model chemistry methods, and with highly accurate MP2 complete basis set limit energies. We find that while all the DFT methods capture the minimum energy structures for tetramers and pentamers, and reproduce the interaction energies well, they fail to find certain structures where London dispersion forces are critical to the interaction. Specifically structures that are not composed of cyclic rings, such as the tetramer pyramid and the pentamer cage structures, are not minima on the DFT potential energy hypersurfaces.
\end{abstract}

Keywords water clusters, water structure, density functionals, MP2, London dispersion forces

\section{INTRODUCTION}

The structure of liquid water is an enigma, and much experimental $^{[1-12]}$ and theoretical ${ }^{[6,13-32]}$ research has been directed at this fundamental problem. ${ }^{[33-40]}$ Classical simulations of water dynamics are only as good as the water models used, with most predicting tetrahedral networks of hydrogen bonding. ${ }^{[14,23,39]}$ In general, water models are developed to reproduce bulk liquid properties, such as the diffusion coefficient, and the heat of vaporization. In contrast to classical treatments, quantum mechanical approaches favor a less

Received 16 April 2007; accepted 2 May 2007.

Acknowledgment is made to NIH, to NSF, and to Hamilton College for support of this work. This project was supported in part by NIH grant 1R15CA115524-01, NSF Grant CHE-0457275, and by NSF grants CHE-0116435 and CHE-0521063 as part of the MERCURY high-performance computer consortium (http:// mercury.chem.hamilton.edu).

Address correspondence to George C. Shields, and Karl N. Kirschner, Department of Chemistry, Center for Molecular Design, Hamilton College, 198 College Hill Road, Clinton, New York 13323, USA. E-mail: gshields@hamilton.edu or kkirschn@hamilton.edu symmetrical tetrahedral configuration of water building blocks. ${ }^{[21]}$

Stepping back from the problem of liquid water, where the experimentally correct answer is a subject of continual debate, studies of gas-phase structures benefit from high-resolution experiments and high-level theoretical calculations. ${ }^{[2,41-58]}$ Xantheas and co-workers have done extensive studies of water clusters using MP2 theory, and have made highly accurate estimates of the MP2 Complete Basis Set (CBS) limit interaction energy. ${ }^{[59-70]}$ These MP2 CBS limit energies can be used as benchmarks for other methods. The search for an appropriate density functional to model hydration has continued since Hillier and co-workers pointed out the problems with existing functionals in 2000. ${ }^{\text {[71] }}$

$\mathrm{Xu}$ and co-workers recently developed an extended hybrid density functional, called X3LYP, with improved descriptions of nonbonded interactions. ${ }^{[72,73]}$ Truhlar and co-workers have developed a new functional, PBE1W, that has been parameterized to yield excellent results for small water clusters composed of three or fewer waters. ${ }^{[74,75]}$ Several groups have made critical evaluations of certain aspects of DFT theory used to model water clusters. ${ }^{[76,77]}$ Johnson and DiLabio have compared six density functionals versus the MP2 method for their ability to predict binding energies for 28 van der Waals dimers, and find that the B971/6$311++\mathrm{G}(3 \mathrm{df}, 3 \mathrm{pd})$ had the best overall performance for binding energies. ${ }^{[78]}$ In this paper we examine the ability of the B971, B3LYP, PBE1W, and X3LYP DFT methods to model the structures and energies of small water clusters, from the dimer up to the octamer, by comparing these methods to the Xantheas' MP2 CBS results.

This investigation was spurred by the desire to find a DFT method that would provide reliable interaction energy prediction for water cluster formation at modest cost. Understanding the thermodynamics of larger water cluster formation becomes increasingly difficult as the cluster size increases, because of the dramatically increased cost in time, using the well-established MP2 correlated ab initio quantum method. If one can predict the general relative configurational stability and thermodynamics of small water clusters using DFT methods, then it 
is possible to study much larger water cluster systems, and have confidence in the results.

\section{METHODS}

We have used the B3LYP method ${ }^{[79,80]}$ with the 6-31 $+\mathrm{G}(\mathrm{d}, 2 \mathrm{p})$ basis set and the PBE1W method with the MG3S basis set ${ }^{[74]}$ to calculate the structures and energies of the minimum energy water dimer, trimer, tetramer, and pentamer structures. For water, the MG3S basis set is identical to the $6-311+\mathrm{G}(2 \mathrm{df}, 2 \mathrm{p})$ basis set. ${ }^{[74]}$ In addition we have used these methods to determine the structures and energies of the Cage, Prism, and Cyclic hexamers, and two Octamers. We have used the CBS-APNO model chemistry method ${ }^{[81]}$ to determine the structure and energies of the $\mathrm{D}_{2 \mathrm{~d}}$ and $\mathrm{S}_{4}$ octamers, which are the two lowest energy octamers. ${ }^{[82]} \mathrm{We}$ used the $\mathrm{G} 3^{[83]}$ and CBS-APNO energies from previous work for the smaller clusters. ${ }^{[82,84-86]}$

In addition we have used the PBE1W/MG3S method ${ }^{[74]}$ and the X3LYP/aug-cc-pVTZ and B971/6-311++G(3df,3pd) methods ${ }^{[72,87]}$ to examine various tetramer and pentamer structures present on the MP2 potential energy hypersurface. ${ }^{[86]}$ Each density functional theory and basis set (i.e., DFT/Basis set) listed above has been previously reported to be an optimal combination for studying water clusters. ${ }^{[73,75,78]}$ Unless otherwise noted, all energies where determined at the same level of theory used for geometry optimization.

Inclusion of the counterpoise correction does not always improve the results, because for moderate or large basis sets there are other sources of error that may be comparable to or larger than the basis set superposition error (BSSE). ${ }^{[76]}$ This seems to be particularly true for water clusters. ${ }^{[76]}$ In addition, the MP2 CBS energies are the same with and without considering BSSE, once extrapolation is performed to arrive at the CBS limit. As we have used large basis sets for the DFT calculations, we have not corrected for BSSE. All calculations were performed using Gaussian 03. ${ }^{[88]}$

\section{RESULTS}

Table 1 contains the results for the B3LYP/aug-cc-pVTZ// B3LYP/6-31G* method, the B3LYP/6-31 + G(d,2p)//B3LYP $/ 6-31+\mathrm{G}(\mathrm{d}, 2 \mathrm{p})$ model, the PBE1W/MG3S//PBE1W/MG3S method, the X3LYP/aug-cc-pVTZ(-f)//X3LYP/aug-ccpVTZ(-f) method, the MP2 CBS estimates of Xantheas, and the G3 and CBS-APNO model chemistry results. ${ }^{[82,86]}$ Figure 1 shows the minima for the water dimer, trimer, tetramer, pentamer, along with three hexamers and two octamers. Figure 2 shows the cyclic $\mathrm{C}_{\mathrm{i}}$ and Pyramid tetramers and the Cage A pentamer.

\section{DISCUSSION}

\section{Structures}

We have previously explored the potential energy hypersurface for the water tetramer and pentamer using MP2 methods. ${ }^{[86]}$ We found five tetramer structures, the lowest energy cyclic $\mathrm{S}_{4}$ (Figure 1), the cyclic $\mathrm{C}_{\mathrm{i}}$, a Pyramid, a Lasso, and a cyclic structure with two waters as double donors and two waters as double acceptors (2DDAA) as minima on the MP2(full)/6-31G* surface. We have shown that the MP2(full)/6-31G* geometries are surprisingly accurate when compared to available experimental and MP2 benchmark calculations. ${ }^{[82,86]}$ Many researchers have investigated water tetramers and further discussion and references can be found in our previous paper. ${ }^{[86]}$

TABLE 1

Comparison of the MP2 complete basis set changes in electronic energies $\left(\Delta \mathrm{E}_{\text {elec }}\right)$ to DFT and model chemistry values for the formation of water clusters

\begin{tabular}{lcccccrr}
\hline Reaction & MP2 CBS $^{a}$ & B3LYP $^{b}$ & B3LYP $^{c}$ & PBE1W $^{d}$ & CBS-APNO $^{2}$ & G3 & X3LYP $^{e}$ \\
\hline $2 \mathrm{H}_{2} \mathrm{O} \rightarrow\left(\mathrm{H}_{2} \mathrm{O}\right)_{2}$ & -4.9 & -2.88 & -5.36 & -5.23 & $-5.07^{f}$ & $-5.14^{f}$ & -5.00 \\
$3 \mathrm{H}_{2} \mathrm{O} \rightarrow\left(\mathrm{H}_{2} \mathrm{O}\right)_{3}$ & -15.8 & -13.39 & -16.24 & -16.04 & $-15.71^{f}$ & $-15.76^{f}$ & -15.52 \\
$4 \mathrm{H}_{2} \mathrm{O} \rightarrow\left(\mathrm{H}_{2} \mathrm{O}\right)_{4}$ & -27.6 & -25.14 & -29.38 & -28.76 & $-27.79^{f}$ & $-28.22^{f}$ & -27.73 \\
$5 \mathrm{H}_{2} \mathrm{O} \rightarrow\left(\mathrm{H}_{2} \mathrm{O}\right)_{5}$ & -36.3 & -33.51 & -38.95 & -38.01 & $-36.77^{f}$ & $-37.26^{f}$ & -36.57 \\
$6 \mathrm{H}_{2} \mathrm{O} \rightarrow \mathrm{Cage}$ & -45.8 & -39.97 & -46.76 & -46.46 & $-46.78^{f}$ & $-47.68^{f}$ & -44.78 \\
$6 \mathrm{H}_{2} \mathrm{O} \rightarrow \mathrm{Prism}$ & -45.9 & -39.69 & -46.56 & -46.39 & $-47.18^{f}$ & $-48.18^{f}$ & -44.69 \\
$6 \mathrm{H}_{2} \mathrm{O} \rightarrow \mathrm{S}_{6}$ & -44.8 & -41.38 & -48.29 & -46.80 & $-45.57^{f}$ & $-45.96^{f}$ & -45.04 \\
$8 \mathrm{H}_{2} \mathrm{O} \rightarrow \mathrm{D}_{2 \mathrm{~d}}$ & -72.7 & -64.60 & -74.41 & -74.02 & -74.76 & -76.41 & -71.05 \\
$8 \mathrm{H}_{2} \mathrm{O} \rightarrow \mathrm{S}_{4}$ & -72.7 & -64.53 & -74.46 & -74.05 & -74.73 & -76.36 & -71.35 \\
\hline
\end{tabular}

${ }^{a}$ MP2 complete basis set limit estimates of Xantheas; References [63, 67, 69]. All energies in kcal/mol.

${ }^{b} \mathrm{~B} 3 \mathrm{LYP} /$ aug-cc-pVTZ//B3LYP/6-31G*.

${ }^{c}$ B3LYP $/ 6-31+\mathrm{G}(\mathrm{d}, 2 \mathrm{p}) / /$ B3LYP/6-31 + G(d,2p).

${ }^{d} \mathrm{PBE} 1 \mathrm{~W} / \mathrm{MG} 3 \mathrm{~S} / / \mathrm{PBE} 1 \mathrm{~W} / \mathrm{MG} 3 \mathrm{~S}$.

${ }^{e}$ X3LYP/aug-cc-pVTZ(-f)//X3LYP/aug-cc-pVTZ(-f); Reference [73].

${ }^{f}$ Reference [84]. 


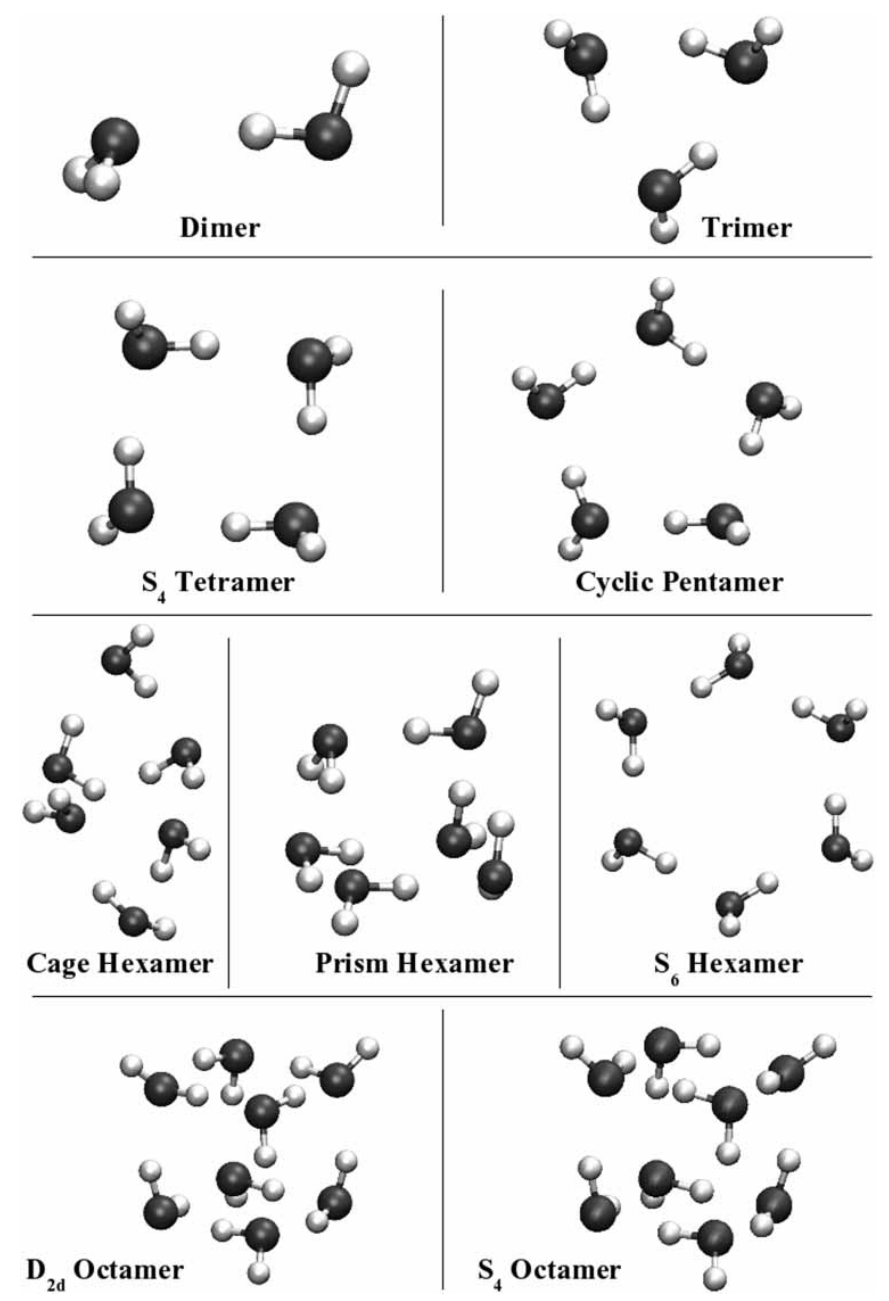

FIG. 1. The structures of the water clusters; the dimer, trimer, tetramer, pentamer, hexamers, and octamers presented in Table 1.

Of particular interest for this work are the cyclic $\mathrm{C}_{\mathrm{i}}$ and the Pyramid tetramer, shown in Figure 2. The cyclic $C_{i}$ structure has inversion symmetry, and is higher in energy than the $S_{4}$ isomer because of greater hydrogen-hydrogen repulsion. The Pyramid has four true hydrogen bonds, two in the bottom of the Pyramid and two connecting the top water to the bottom of the Pyramid. The relevant bond angles are 156 and 149

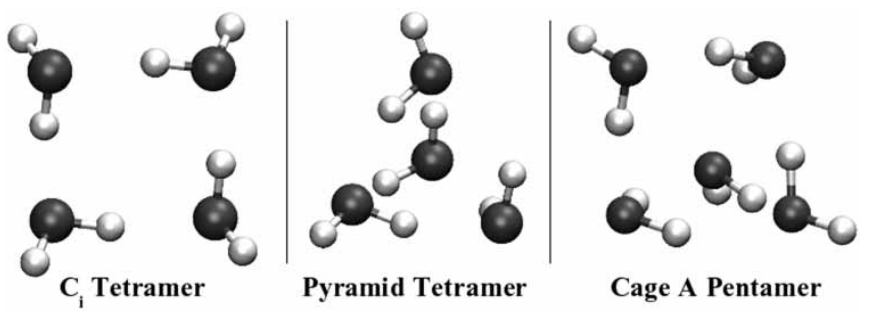

FIG. 2. The structures of the cyclic $\mathrm{C}_{\mathrm{i}}$ and pyramid tetramers, and the Cage $\mathrm{A}$ pentamer. degrees on the bottom of the Pyramid and 147 and 150 degrees connecting the top water to the Pyramid. In addition the Pyramid has an apparent hydrogen bond distance of 2.36 $\AA$ in the bottom of the Pyramid, and an apparent hydrogen bond distance of $2.440 \AA$ connecting the top water to the bottom of the Pyramid.

The relevant apparent hydrogen bond angles are 123 degrees on the bottom of the Pyramid and 119 degrees connecting the top water to the Pyramid. These apparent hydrogen bonds are better described as London dispersion forces. Thus a mixture of four hydrogen bonds and London dispersion forces (the apparent hydrogen bonds) holds the Pyramid together. The Pyramid structure is a minimum on the MP2 surface, which we confirmed by increasing the basis set size to aug-cc-pVDZ and re-optimizing the structure.

To test the ability of DFT to replicate selected points on the MP2 potential energy hypersurface we have selected three of the best DFT methods (PBE1W, B971, and X3LYP) for use with water clusters. We have also included the B3LYP method, which is the most used functional, and generally obtains good results for a wide range of systems. ${ }^{[89]}$ For each functional we have used the basis sets that have been determined to be optimal for water clusters, which is crucial for maximizing the performance of each functional. ${ }^{[73,75,78]}$

We used each method with its optimal basis set to optimize the Pyramid structure. All four methods, B3LYP/6$31+\mathrm{G}(\mathrm{d}, 2 \mathrm{p})$, PBE1W/MG3S, X3LYP/aug-cc-pVTZ, and B971/6-311++G(3df,3pd), fail to locate the Pyramid structure on the DFT potential energy hypersurface. The B3LYP and X3LYP methods convert the Pyramid to the cyclic $\mathrm{S}_{4}$ minimum (Figure 1), while the B971 and PBE1W methods optimize to the cyclic $\mathrm{C}_{\mathrm{i}}$ minima (Figure 2). Changing the step size in geometry optimization in Gaussian03 to $1 / 30$ of the default size has no effect on the final results of the optimization process.

For the water pentamer, G3 model chemistry, which uses MP2/6-31G* minima structures to obtain electronic energies, was previously used to locate 10 unique pentamers. ${ }^{[86]}$ The lowest energy structure is cyclic, and there are two sets of similar configurations. The MP2 method finds five fused ring structures and four cage structures. The fused ring structures can be visualized as a tetramer fused with a trimer (labeled $\mathrm{A}, \mathrm{B}$, and $\mathrm{C}$ in reference ${ }^{[86]}$ ) or as the fusion of two trimers (labeled D and $\mathrm{E}$ in reference ${ }^{[86]}$ ). The cage structures do not contain any fully formed cyclic units, but rather form a cage from the constituent waters, as exemplified by Cage A shown in Figure 2.

The four DFT methods have been used to optimize the two representatives of the two sets of similar configurations, the Fused Ring A structure and the Cage A structure. ${ }^{[86]}$ In this case the four DFT methods retain the fused ring motif, but not the cage motif. The four DFT methods transform the Cage A structure to the cyclic minimum. MP2/aug-cc-pVDZ 
geometry optimizations from the same starting structure confirm that the Cage A isomer is a minimum.

We used the PBE1W functional to optimize Cage B, Cage C, Cage D, Fused Ring B, Fused Ring C, Fused Ring D, and Fused Ring $E{ }^{[86]}$ and in these cases the caged structures were optimized to the cyclic minima while the fused ring structures retained their original configuration. Using a maximum step size of $1 / 30$ the default in Gaussian03, or 0.01 Bohr, and computing the force constants at every step, opt $=(\operatorname{maxstep}=1$, calcall $)$, does not change the result for the Cage A or Cage B structure. The Cage C structure does converge as a minimum on the $\mathrm{PBE} 1 \mathrm{~W} / \mathrm{MG} 3 \mathrm{~S}$ potential energy hypersurface, while the Cage D structure converts to an ice-like structure with one central water molecule hydrogen bonded to four surrounding waters in a roughly tetrahedral arrangement.

While it is reassuring that the DFT methods find cyclic structures as minima, the failure to locate the Pyramid tetramer and the Cage A pentamer means that we lack confidence that all of the larger water cluster minima will be located on the DFT potential energy hypersurfaces. Since we do not know, a priori, what structures will be minima when we have more than 10 water molecules, we can not rely on current density functionals to yield accurate results. We are reduced to remembering that density functional theory is a parameterized approach. ${ }^{[89]}$

The PM3 semiempirical approach is an example of a fast method, that is generally good for hydrogen bonded structures, but not energies, ${ }^{[90]}$ and has been used to investigate large water clusters. ${ }^{[91,92]}$ DFT methods are not variationally consistent; so that the use of a large basis set does not guarantee more accurate results, as exemplified in Table 1. It is more prudent to consider each DFT method and basis set combination as a model chemistry, and it is essential to benchmark a given DFT method and basis set for the particular system being studied. ${ }^{[8]}$ For water clusters such benchmarking should ensure that the intermolecular forces present are being modeled accurately.

In this study we have found that the four DFT methods can locate all structures on the potential energy hypersurface where well-formed hydrogen bonds exist. The strong hydrogen-bond forces hold the hexamer structures together. However, when a structure has greatly distorted hydrogen bonds, and/or has putative hydrogen bond distances that exceed $2 \AA$ and putative hydrogen bond angles less than 140 degrees, then the waters are bound together by a combination of weaker hydrogen bonds and London dispersion forces. These DFT methods are then unable to locate these minima, such as the Pyramid tetramer and Cage pentamer structures.

\section{Energies}

The energies of the DFT methods are pretty good when using the appropriate optimal basis sets. It was interesting to observe that calculating energies with the B3LYP/
aug-cc-pVTZ basis set using B3LYP/6-31G* geometries were much poorer than for the total structural and energy calculations at the B3LYP/6-31 + G(d,2p) level. Previous work in our lab on ion clusters revealed that the B3LYP/aug-ccpVTZ//B3LYP/6-31G* method gave excellent results compared to experiment for $\mathrm{NH}_{4}^{+}\left(\mathrm{H}_{2} \mathrm{O}\right)_{\mathrm{n}}$ and $\mathrm{NH}_{4}^{+}\left(\mathrm{NH}_{3}\right)_{\mathrm{n}}$ clusters $(n=1-4) .{ }^{[93]}$ Good as these results are for ion neutral hydrogen bonding, this method fails to be quantitative for neutral water clusters. Thus, while the augmented correlation consistent basis set can be used with B3LYP to avoid more expensive quantum chemical methods (i.e., MP2) for ion-water clusters, ${ }^{[94]}$ this procedure is not useful for neutral water clusters.

The fact that certain basis sets are optimal for certain functionals shows the empirical nature of DFT. The PBE1W/ MG3S method is better than the G3 model chemistry, and as good as the CBS-APNO model chemistry, when compared against the MP2 CBS limit. The X3LYP/aug-cc-pVTZ(-f) method is even better. These later two DFT methods yield accurate energies, and can be trusted as long as the water clusters that are being investigated are composed of cyclic rings. The big caveat on these DFT functionals is that there is no way to ensure that all the minima for larger water clusters have been located.

\section{London Dispersion Forces}

Why do the B3LYP, X3LYP, PBE1W, and B971 DFT methods accurately model cyclic water structures and larger clusters built from cyclic motifs, yet fail to locate more complicated structures such as the Pyramid tetramer and the Cage A pentamer? The answer appears to be that these new functionals, good as they are, still cannot capture dispersion in molecular systems with extensive electron delocalization. While classical hydrogen bonding is well described, more complicated systems that include London dispersion forces are much harder to model. Parameterization can be used to add in dispersion, ${ }^{[95]}$ but as of today it does not appear that any functional incorporates the physics correctly. ${ }^{[78]}$

It has been shown that X3LYP, while accurately describing the hydrogen bonding between nucleic acid base pairs, fails to model stacking interactions. ${ }^{[96]}$ It is well known that stacking interactions are the main stabilizing force for nucleic acid helices, ${ }^{[97-99]}$ but reproducing these forces requires highlevel quantum calculations that include electron correlation. ${ }^{[100,101]}$ Unfortunately, X3LYP does not model London dispersion forces for delocalized molecular systems, and as a consequence, all of the stacked nucleic acid base pairs optimized to planar hydrogen bonded pairs. ${ }^{[96]}$ DFT was only useful to describe stacking when an empirical London dispersion energy term was added. ${ }^{[96]}$ The authors note that given the good performance of X3LYP for $\mathrm{He}$ and $\mathrm{Ne}$ dimers, the London dispersion forces between aromatic systems presumably originate from collective electron delocalization. ${ }^{[96]}$ 
A related problem to capturing all the stationary points on a potential energy hypersurface is to correctly characterize the curvature of the surface at these points. Tschumper's group has investigated this problem, examining 10 stationary points on the water dimer potential energy hypersuface with ten density functional methods. They find that correctly characterizing the nature of the stationary points is far more difficult than determining the energies, and they show that X3LYP, B3LYP, and B971 get the energies right, but fail to reproduce the correct number of imaginary frequencies produced by $\operatorname{CCSD}(\mathrm{T})$ calculations. ${ }^{[77]}$ This problem of characterizing the curvature for the water dimer surface will only become more difficult for larger water clusters that have more complicated potential energy hypersurfaces.

A successful density functional will have to describe molecular systems with delocalized electrons, similar to the water tetramer pyramid structure, and the water pentamer cage structures. Benchmarking new DFT models against systems that can only be described accurately by MP2 and $\operatorname{CCSD}(\mathrm{T})$ correlated ab initio calculations will be necessary before the computational community can have complete confidence in the use of DFT for water clusters. It is not clear to us whether the failure of these DFT functionals to describe the entire gas-phase potential energy hypersurface of the tetramers and pentamers means that these functionals cannot be trusted for liquid water simulations. Since the structure of liquid water is still a subject of much debate, and since the local structure no doubt changes in situations of high pressure, or close confinement, we suggest that investigators proceed with caution, and not over-interpret computational predictions based on DFT.

\section{CONCLUSIONS}

We have tested the B3LYP, X3LYP, PBE1W, and B971 density functionals for their ability to replicate the structures and energies of small water clusters. We find that while all the DFT methods capture the minimum energy structures for tetramers and pentamers, and reproduce the energies well, they fail to find certain structures where London dispersion forces are critical to the overall shape. Specifically, structures that are not composed of cyclic rings, such as the tetramer Pyramid and the pentamer cage structures, are not minima on the DFT potential energy hypersurfaces. Continued work on DFT should focus on capturing London dispersion forces resulting from the electron delocalization in aromatic and other molecular systems with delocalized electrons.

\section{REFERENCES}

1. S. Woutersen, U. Emmerichs, and H.J. Bakker, Femtosecond Mid-IR Pump-probe Spectroscopy of Liquid Water: Evidence for a Two-component Structure, Science, 1997, 278 (5338), $658-660$.
2. F. Keutsch and R.J. Saykally, Water Clusters: Untangling the Mysteries of the Liquid, One Molecule at a Time, Proc. Natl. Acad. Sci., 2001, 98 (19), 10533-10540.

3. M.F. Kropman and H.J. Bakker, Dynamics of Water Molecules in Aqueous Solvation Shells, Science, 2001, 291 (5511), 2118-2120.

4. C.-Y. Ruan, V.A. Lobastov, F. Vigliotti, S. Chen, and A.H. Zewail, Ultrafast Electron Crystallography of Interfacial Water, Science, 2004, 304 (5667), 80-84.

5. S. Myneni, Y. Luo, L.A. Näslund, M. Cavalleri, L. Ojamäe, H. Ogasawara, A. Pelmenschikov, P. Wernet, P. Vaterlein, C. Heske, Z. Hussain, L.G.M. Pettersson, and A. Nilsson, Spectroscopic Probing of Local Hydrogen-bonding Structures in Liquid Water, J. Phys. Cond. Matt., 2002, 14 (8), L213-L219.

6. H.J. Bakker, H.K. Nienhuys, G. Gallot, N. Lascoux, G.M. Gale, J.C. Leicknam, and S. Bratos, Transient Absorption of Vibrationally Excited Water, J. Chem. Phys., 2002, 116 (6), 2592-2598.

7. P. Wernet, D. Nordlund, U. Bergmann, M. Cavalleri, M. Odelius, H. Ogasawara, L.A. Näslund, T.K. Hirsch, L. Ojamäe, P. Glatzel, L.G. M. Pettersson, and A. Nilsson, The Structure of the First Coordination Shell in Liquid Water, Science, 2004, 304 (5673), 995-999.

8. J.B. Brubach, A. Mermet, A. Filabozzi, A. Gerschel, and P. Roy, Signatures of the Hydrogen Bonding in the Infrared Bands of Water, J. Chem. Phys., 2005, 122 (18).

9. L.A. Näslund, J. Lüning, Y. Ufuktepe, H. Ogasawara, P. Wernet, U. Bergmann, L.G.M. Pettersson, and A. Nilsson, X-ray Absorption Spectroscopy Measurements of Liquid Water, J. Phys Chem. B, 2005, 109 (28), 13835-13839.

10. G. Öhrwall, R.F. Fink, M. Tchaplyguine, L. Ojamäe, M. Lundwall, R.R.T. Marinho, A.N. de Brito, S.L. Sorensen, M. Gisselbrecht, R. Feifel, T. Rander, A. Lindblad, J. Schulz, L.J. Saethre, N. Mårtensson, S. Svensson, and O. Björneholm, The Electronic Structure of Free Water Clusters Probed by Auger Electron Spectroscopy, J. Chem. Phys., 2005, 123 (5).

11. M. Hakala, K. Nygard, S. Manninen, L.G.M. Pettersson, and K. Hamalainen, Intra- and Intermolecular Effects in the Compton Profile of Water, Phys. Rev. B, 2006, 73 (3).

12. N.K. Alphonse, S.R. Dillon, R.C. Dougherty, D.K. Galligan, and L.N. Howard, Direct Raman Evidence for a Weak Continuous Phase Transition in Liquid Water, J. Phys. Chem. A, 2006, 110, 7577-7580.

13. S.W. Benson and E.D. Siebert, A Simple 2-structure Model for Liquid Water, J. Am. Chem. Soc., 1992, 114 (11), 4269-4276.

14. P.L. Silvestrelli and M. Parrinello, Structural, Electronic, and Bonding Properties of Liquid Water from First Principles, J. Chem. Phys., 1999, 111 (8), 3572-3580.

15. P.L. Geissler, C. Dellago, D. Chandler, J. Hutter, and M. Parrinello, Autoionization in Liquid Water, Science, 2001, 291 (5511), 2121-2124.

16. S. Izvekov and G.A. Voth, Car-Parrinello Molecular Dynamics Simulation of Liquid Water: New Results, J. Chem. Phys., 2002, 116 (23), 10372-10376.

17. B. Chen, I. Ivanov, M.L. Klein, and M. Parrinello, Hydrogen Bonding in Water, Phys. Rev. Lett., 2003, 91 (21).

18. D. Asthagiri, L.R. Pratt, and J.D. Kress, Free Energy of Liquid Water on the Basis of Quasichemical Theory and ab initio 
Molecular Dynamics, Phys. Rev. E: Stat. Nonlin. Soft Matter Phys., 2003, 68 (4-1).

19. J.C. Grossman, E. Schwegler, E.W. Draeger, F. Gygi, and G. Galli, Towards an Assessment of the Accuracy of Density Functional Theory for First Principles Simulations of Water, J. Chem. Phys., 2004, 120 (1), 300-311.

20. M. Allesch, E. Schwegler, F. Gygi, and G. Galli, A First Principles Simulation of Rigid Water, J. Chem. Phys., 2004, 120 (11), 5192-5198.

21. L.H. de la Pena, M.S. G. Razul, and P.G. Kusalik, Impacts of Quantization on the Properties of Liquid Water, J. Phys. Chem. A, 2005, 109 (32), 7236-7241.

22. V. Buch, Molecular Structure and $\mathrm{OH}$-stretch Spectra of Liquid Water Surface, J. Phys Chem. B, 2005, 109 (38), 17771-17774.

23. A.K. Soper, An Asymmetric Model for Water Structure, J. Phys. Cond. Matter, 2005, 17 (45), S3273-S3282.

24. A.M. Lindenberg, Y. Acremann, D.P. Lowney, P.A. Heimann, T.K. Allison, T. Matthews, and R.W. Falcone, Time-resolved Measurements of the Structure of Water at Constant Density, J. Chem. Phys., 2005, 122 (20).

25. D. Prendergast, J.C. Grossman, and G. Galli, The Electronic Structure of Liquid Water within Density-functional Theory, J. Chem. Phys., 2005, 123 (1).

26. P.C. do Couto, S.G. Estacio, and B.J.C. Cabral, The Kohn-Sham Density of States and Band Gap of Water: From Small Clusters to Liquid Water, J. Chem. Phys., 2005, 123 (5).

27. T.L. Jansen, T. Hayashi, W. Zhuang, and S. Mukamel, Stochastic Liouville Equations for Hydrogen-bonding Fluctuations and their Signatures in Two-dimensional Vibrational Spectroscopy of Water, J. Chem. Phys., 2005, 123 (11).

28. T. Todorova, A.P. Seitsonen, J. Hutter, I.F.W. Kuo, and C.J. Mundy, Molecular Dynamics Simulation of Liquid Water: Hybrid Density Functionals, J. Phys Chem. B, 2006, 110 (8), 3685-3691.

29. G.S. Fanourgakis and S.S. Xantheas, The Flexible, Polarizable, Thole-type Interaction Potential for Water (TTM2-F) Revisited, J. Phys. Chem. A, 2006, 110 (11), 4100-4106.

30. M. Odelius, M. Cavalleri, A. Nilsson, and L.G. M. Pettersson, Xray Absorption Spectrum of Liquid Water from Molecular Dynamics Simulations: Asymmetric Model, Phys. Rev. B, 2006, 73 (2).

31. B. Winter and M. Faubel, Photoemission from Liquid Aqueous Solutions, Chem. Rev., 2006, 106 (4), 1176-1211.

32. D. Laage and J.T. Hynes, A Molecular Jump Mechanism of Water Reorientation, Science, 2006, 311 (5762), 832-835.

33. R. Ludwig, Water: from Clusters to the Bulk, Angew. Chem. Int. Ed., 2001, 40, 1808-1827.

34. G. Bertrand, A Reappraisal of what we have Learnt During Three Decades of Computer Simulations on Water, J. Mol. Liq., 2002, 101 (1-3), 219-260.

35. T. Head-Gordon and G. Hura, Water Structure from Scattering Experiments and Simulation, Chem. Rev., 2002, 102, 2651-2670.

36. G. Hura, D. Russo, R.M. Glaeser, T. Head-Gordon, M. Krack, and M. Parrinello, Water Structure as a Function of Temperature from X-ray Scattering Experiments and ab initio Molecular Dynamics, PCCP Phys. Chem. Chem. Phys., 2003, 5 (10), $1981-1991$.
37. J.D. Eaves, J.J. Loparo, C.J. Fecko, S.T. Roberts, A. Tokmakoff, and P.L. Geissler, Hydrogen Bonds in Liquid Water are Broken Only Fleetingly, Proc. Natl. Acad. Sci., 2005, 102 (37), 13019-13022.

38. J.D. Smith, C.D. Cappa, K.R. Wilson, R.C. Cohen, P.L. Geissler, and R.J. Saykally, Unified Description of Temperature-dependent Hydrogen-bond Rearrangements in Liquid Water, Proc. Natl. Acad. Sci., 2005, 102 (40), 14171-14174.

39. Y.A. Mantz, B. Chen, and G.J. Martyna, Structural Correlations and Motifs in Liquid Water at Selected Temperatures: Ab initio and Empirical Model Predictions, J. Phys Chem. B, 2006, 110 (8), 3540-3554.

40. T. Head-Gordon and M.E. Johnson, Tetrahedral Structure of Chains of Liquid Water, Proc. Natl. Acad. Sci., 2006, 103 (21), 7973-7977.

41. K. Kim, K.D. Jordan, and T.S. Zwier, Low-energy Structures and Vibrational Frequencies of the Water Hexamer: Comparison with Benzene- $\left(\mathrm{H}_{2} \mathrm{O}\right)_{6}$, J. Am. Chem. Soc., 1994, 116 (25), $11568-11569$.

42. K. Kim and K.D. Jordan, Comparison of Density Functional and MP2 Calculations on the Water Monomer and Dimer, J. Phys. Chem., 1994, 98, 10089-10094.

43. J.M. Pedulla, F. Villa, and K.D. Jordan, Binding Energy of the Ring Form of $\left(\mathrm{H}_{2} \mathrm{O}\right)_{6}$ : Comparison of the Predictions of Conventional and Localized-orbital MP2 Calculations, J. Chem. Phys., 1996, 105 (24), 11091-11099.

44. K. Liu, M.G. Brown, C. Carter, R.J. Saykally, J.K. Gregory, and D.C. Clary, Characterization of a Cage Form of the Water Hexamer, Nature, 1996, 381 (6582), 501-503.

45. J.K. Gregory, D.C. Clary, K. Liu, M.G. Brown, and R.J. Saykally, The Water Dipole Moment in Water Clusters, Science, 1997, 275, 814-817.

46. S.Y. Fredericks, J.M. Pedulla, K.D. Jordan, and T.S. Zwier, OH Stretch IR Spectra of $\left(\mathrm{H}_{2} \mathrm{O}\right)_{3}$ and Benzene- $\left(\mathrm{H}_{2} \mathrm{O}\right)_{3}$, Theor. Chem. Acc., 1997, 96 (1), 5-55.

47. C.J. Gruenloh, J.R. Carney, C.A. Arrington, T.S. Zwier, S.Y. Fredericks, and K.D. Jordan, Infrared Spectrum of a Molecular Ice Cube: the $S_{4}$ and $D_{2 d}$ Water Octamers in Benzene(water) 8 , Science, 1997, 276, 1678-1681.

48. C.J. Gruenloh, J.R. Carney, F.C. Hagemeister, C.A. Arrington, T.S. Zwier, S.Y. Fredericks, J.T. Wood, and K.D. Jordan, Resonant Ion-dip Infrared Spectroscopy of the $S_{4}$ and $D_{2 d}$ Wafer Octamers in Benzene-(water)(8) and Benzene ${ }_{2}$-(water) $)_{8}$, J. Chem. Phys., 1998, 109 (16), 6601-6614.

49. J.M. Pedulla, H. Kim, and K.D. Jordan, Theoretical Study of the n-body Interaction Energies of the Ring, Cage and Prism Forms of $\left(\mathrm{H}_{2} \mathrm{O}\right)_{6}$, Chem. Phys. Lett., 1998, 291, 78-84.

50. J.M. Pedulla and K.D. Jordan, Accurate Calculation of the Interaction energies in hydrogen-bonded Complexes, In Recent Theoretical and Experimental Advances in Hydrogen Bonded Clusters, S.S. Xantheas (ed.), Kluwer Academic Publishers, Netherlands, 2000, 435-44.

51. N. Goldman, R.S. Fellers, C. Leforestier, and R.J. Saykally, Water Dimers in the Atmosphere: Equilibrium Constant for Water Dimerization from the VRT(ASP-W) Potential Surface, J. Phys. Chem. A, 2001, 105 (3), 515-519.

52. F.N. Keutsch, J.D. Cruzan, and R.J. Saykally, The Water Trimer, Chem. Rev., 2003, 103 (7), 2533-2577. 
53. A.J. Huneycutt and R.J. Saykally, Building Solutions-One Molecule at a Time, Science, 2003, 299, 1329-1330.

54. J.M. Headrick, E.G. Diken, R.S. Walters, N.I. Hammer, R.A. Christie, J. Cui, E.M. Myshakin, M.A. Duncan, M.A. Johnson, and K.D. Jordan, Spectral Signatures of Hydrated Proton Vibrations in Water Clusters, Science, 2005, 308 (5729), 1765-1769.

55. B.C. Garrett, D.A. Dixon, D.M. Camaioni, D.M. Chipman, M.A. Johnson, C.D. Jonah, G.A. Kimmel, J.H. Miller, T.N. Rescigno, P.J. Rossky, S.S. Xantheas, S.D. Colson, A.H. Laufer, D. Ray, P.F. Barbara, D.M. Bartels, K.H. Becker, H. Bowen, S.E. Bradforth, I. Carmichael, J.V. Coe, L.R. Corrales, J.P. Cowin, M. Dupuis, K.B. Eisenthal, J.A. Franz, M.S. Gutowski, K.D. Jordan, B.D. Kay, J.A. LaVerne, S.V. Lymar, T.E. Madey, C.W. McCurdy, D. Meisel, S. Mukamel, A.R. Nilsson, T.M. Orlando, N.G. Petrik, S.M. Pimblott, J.R. Rustad, G.K. Schenter, S.J. Singer, A. Tokmakoff, L.S. Wang, C. Wittig, and T.S. Zwier, Role of Water in Electron-initiated Processes and Radical Chemistry: Issues and Scientific Advances, Chem. Rev., 2005, 105 (1), 355-389.

56. K.S. Alongi, T.S. Dibble, G.C. Shields, and K.N. Kirschner, Exploration of the Potential Energy Surfaces, Prediction of Atmospheric Concentrations, and Prediction of Vibrational Spectra for the $\mathrm{HO}_{2} \cdot \cdot\left(\mathrm{H}_{2} \mathrm{O}\right)_{\mathrm{n}}(\mathrm{n}=1-2)$ Hydrogen Bonded Complexes, J. Phys. Chem. A, 2006, 110 (10), 3686-3691.

57. M.E. Dunn, T.M. Evans, K.N. Kirschner, and G.C. Shields, Prediction of Accurate Anharmonic Experimental Vibrational Frequencies for Water Clusters, $\left(\mathrm{H}_{2} \mathrm{O}\right)_{(\mathrm{n})}, \mathrm{n}=2-5$, J. Phys. Chem. A, 2006, 110 (1), 303-309.

58. K.N. Kirschner, G.M. Hartt, T.M. Evans, and G.C. Shields, In Search of $\mathrm{CS}_{2}\left(\mathrm{H}_{2} \mathrm{O}\right)_{\mathrm{n}}=1-4$ Clusters, J. Chem. Phys., 2007, $126,154320$.

59. S.S. Xantheas and T.H. Dunning, Ab initio Studies of Cyclic Water Clusters $\left(\mathrm{H}_{2} \mathrm{O}\right)_{\mathrm{n}}, \mathrm{n}=\mathrm{I}-6.1$. Optimal Structures and Vibrational-spectra, J. Chem. Phys., 1993, 99 (11), 8774-8792.

60. S.S. Xantheas and T.H. Dunning, The Structure of the Water Trimer from ab initio Calculations, J. Chem. Phys., 1993, 98 (10), 8037-8040.

61. S.S. Xantheas, Ab initio Studies of Cyclic Water Clusters $\left(\mathrm{H}_{2} \mathrm{O}\right)_{\mathrm{n}}, \mathrm{n}=1-6$. II. Analysis of Many-body Interactions, J. Chem. Phys., 1994, 100 (10), 7523-7534.

62. S.S. Xantheas, Ab initio Studies of Cyclic Water Clusters $\left(\mathrm{H}_{2} \mathrm{O}\right)_{n}, \mathrm{n}=1-6$. III. Comparison of Density Functional with MP2 Results, J. Chem. Phys., 1995, 102 (11), 4505-4517.

63. S.S. Xantheas, On the Importance of the Fragment Relaxation Energy Terms in the Estimation of the Basis Set Superposition Error Correction to the Intermolecular Interaction Energy, J. Chem. Phys., 1996, 104 (21), 8821-8824.

64. S.S. Xantheas, Cooperativity and Hydrogen Bonding Network in Water Clusters, Chem. Phys., 2000, 258 (2-3), 225-231.

65. C.J. Burnham and S.S. Xantheas, Development of Transferable Interaction Models for Water. I. Prominent Features of the Water Dimer Potential Energy Surface, J. Chem. Phys., 2002, 116 (4), 1479-1492.

66. C.J. Burnham, S.S. Xantheas, M.A. Miller, B.E. Applegate, and R.E. Miller, The Formation of Cyclic Water Complexes by
Sequential Ring Insertion: Experiment and Theory, J. Chem. Phys., 2002, 117 (3), 1109-1122.

67. S.S. Xantheas, C.J. Burnham, and R.J. Harrison, Development of Transferable Interaction Models for Water. II. Accurate Energetics of the First Few Water Clusters from First Principles, J. Chem. Phys., 2002, 116 (4), 1493-1499.

68. C.J. Burnham and S.S. Xantheas, Development of Transferable Interaction Models for Water. IV. A Flexible, All-atom Polarizable Potential (TTM2-F) Based on Geometry Dependent Charges Derived from an ab initio Monomer Dipole Moment Surface, J. Chem. Phys., 2002, 116 (12), 5115-5124.

69. S.S. Xantheas and E. Apra, The Binding Energies of the $D_{2 d}$ and $\mathrm{S}_{4}$ Water Octamer Isomers: High-level Electronic Structure and Empirical Potential Results, J. Chem. Phys., 2004, 120 (2), $823-828$.

70. S.S. Xantheas, Interaction Potentials for Water from Accurate Cluster Calculations, Intermolecular Forces and Clusters II, 2005, 116, 119-148.

71. R.J. Hall, I.H. Hillier, and M.A. Vincent, Which Density Functional Should be Used to Model Hydration? Chem. Phys. Lett., 2000, 320 (1-2), 139-143.

72. X. Xu, Q.S. Zhang, R.P. Muller, and W.A. Goddard, An Extended Hybrid Density Functional (X3LYP) with Improved Descriptions of Nonbond Interactions and Thermodynamic Properties of Molecular Systems, J. Chem. Phys., 2005, 122 (1).

73. J.T. Su, X. Xu, and W.A. Goddard, Accurate Energies and Structures for Large Water Clusters Using the X3LYP Hybrid Density Functional, J. Phys. Chem. A, 2004, 108 (47), 10518-10526.

74. E.E. Dahlke and D.G. Truhlar, Improved Density Functionals for Water, J. Phys Chem. B, 2005, 109 (33), 15677-15683.

75. E.E. Dahlke and D.G. Truhlar, Assessment of the Pairwise Additive Approximation and Evaluation of Many-body Terms for Water Clusters, J. Phys Chem. B, 2006, 110 (22), 10595-10601.

76. G.I. Csonka, A. Ruzsinszky, and J.P. Perdew, Proper Gaussian Basis Sets for Density Functional Studies of Water Dimers and Trimers, J. Phys Chem. B, 2005, 109 (46), 21471-21475.

77. J.A. Anderson and G.S. Tschumper, Characterizing the Potential Energy Surface of the Water Dimer with DFT: Failures of Some Popular Functionals for Hydrogen Bonding, J. Phys. Chem. A, 2006, 110 (22), 7268-7271.

78. E.R. Johnson and G.A. DiLabio, Structure and Binding Energies in van der Waals Dimers: Comparison between Density Functional Theory and Correlated ab initio Methods, Chem. Phys. Lett., 2006, 419 (4-6), 333-339.

79. C.T. Lee, W.T. Yang, and R.G. Parr, Development of the ColleSalvetti Correlation-energy Formula into a Functional of the Electron-density, Phys. Rev. B, 1988, 37 (2), 785-789.

80. A.D. Becke, Density-functional Thermochemistry. 3. The Role of Exact Exchange, J. Chem. Phys., 1993, 98 (7), 5648-5652.

81. J.A. Montgomery, J.W. Ochterski, and G.A. Petersson, A Complete Basis-set Model Chemistry. 4. An Improved Atomic Pair Natural Orbital Method, J. Chem. Phys., 1994, 101 (7), 5900-5909.

82. M.B. Day, K.N. Kirschner, and G.C. Shields, Pople's Gaussian-3 Model Chemistry Applied to an Investigation of $\left(\mathrm{H}_{2} \mathrm{O}\right)_{8}$ Water Clusters, Int. J. Quantum Chem., 2005, 102 (5), 565-572. 
83. L.A. Curtiss, K. Raghavachari, P.C. Redfern, V. Rassolov, and J.A. Pople, Gaussian-3 (G3) Theory for Molecules Containing First and Second-row Atoms, J. Chem. Phys., 1998, 109 (18), 7764-7776.

84. M.E. Dunn, E.K. Pokon, and G.C. Shields, Thermodynamics of Forming Water Clusters at Various Temperatures and Pressures by Gaussian-2, Gaussian-3, Complete Basis Set-QB3, and Complete Basis Set-APNO Model Chemistries; Implications for Atmospheric Chemistry, J. Am. Chem. Soc., 2004, 126 (8), 2647-2653.

85. M.E. Dunn, E.K. Pokon, and G.C. Shields, The Ability of the Gaussian-2, Gaussian-3, Complete Basis Set-QB3, and Complete Basis Set-APNO Model Chemistries to Model the Geometries of Small Water Clusters, Int. J. Quantum Chem., 2004, 100 (6), 1065-1070.

86. M.B. Day, K.N. Kirschner, and G.C. Shields, Global Search for Minimum Energy $\left(\mathrm{H}_{2} \mathrm{O}\right)_{n}$ Clusters, $\mathrm{n}=3-5$, J. Phys. Chem. A, 2005, 109 (30), 6773-6778.

87. F.A. Hamprecht, A.J. Cohen, D.J. Tozer, and N.C. Handy, Development and Assessment of New Exchange-correlation Functionals, J. Chem. Phys., 1998, 109 (15), 6264-6271.

88. M.J. Frisch, G.W. Trucks, H.B. Schlegel, G.E. Scuseria, M.A. Robb, J.R. Cheeseman, J.A. Montgomery, T. Vreven, K.N. Kudin, J.C. Burant, J.M. Millam, S.S. Iyengar, J. Tomasi, V. Barone, B. Mennucci, M. Cossi, G. Scalmani, N. Rega, G.A. Petersson, H. Nakatsuji, M. Hada, M. Ehara, K. Toyota, R. Fukuda, J. Hasegawa, M. Ishida, T. Nakajima, Y. Honda, O. Kitao, H. Nakai, M. Klene, X. Li, J.E. Knox, H.P. Hratchian, J.B. Cross, V. Bakken, C. Adamo, J. Jaramillo, R. Gomperts, R.E. Stratmann, O. Yazyev, A.J. Austin, R. Cammi, C. Pomelli, J.W. Ochterski, P.Y. Ayala, K. Morokuma, G.A. Voth, P. Salvador, J.J. Dannenberg, V.G. Zakrzewski, S. Dapprich, A.D. Daniels, M.C. Strain, O. Farkas, D.K. Malick, A.D. Rabuck, K. Raghavachari, J.B. Foresman, J.V. Ortiz, Q. Cui, A.G. Baboul, S. Clifford, J. Cioslowski, B.B. Stefanov, G. Liu, A. Liashenko, P. Piskorz, I. Komaromi, R.L. Martin, D.J. Fox, T. Keith, M.A. Al-Laham, C.Y. Peng, A. Nanayakkara, M. Challacombe, P.M. W. Gill, B. Johnson, W. Chen, M.W. Wong, C. Gonzalez, and J.A. Pople, Gaussian 03 (Revision D.01), 2004, Gaussian, Inc., Wallingford, CT.

89. C.J. Cramer, Essentials of Computational Chemistry: Theories and Models, 2nd edn., 2004, John Wiley \& Sons, Inc., New York.

90. M.W. Jurema and G.C. Shields, Ability of the PM3 Quantum Mechanical Method to Model inter Molecular Hydrogen
Bonding Between Neutral Molecules, J. Comput. Chem., 1993, 14 (1), 89-104.

91. M.W. Jurema, K.N. Kirschner, and G.C. Shields, Modeling of Magic Water Clusters $\left(\mathrm{H}_{2} \mathrm{O}\right)_{20}$ and $\left(\mathrm{H}_{2} \mathrm{O}\right)_{21} \mathrm{H}^{+}$with the PM3 Quantum-mechanical Method, J. Comput. Chem., 1993, 14 (11), 1326-332.

92. K.N. Kirschner and G.C. Shields, Quantum-mechanical Investigation of Large Water Clusters, Int. J. Quantum Chem., 1994, 28, 349-360.

93. F.C. Pickard, M.E. Dunn, and G.C. Shields, Comparison of Model Chemistry and Density Functional Theory Thermochemical Predictions with Experiment for Formation of Ionic Clusters of the Ammonium Cation Complexed with Water and Ammonia; Atmospheric Implications, J. Phys. Chem. A, 2005, 109 (22), 4905-4910.

94. F.C. Pickard, E.K. Pokon, M.D. Liptak, and G.C. Shields, Comparison of CBS-QB3, CBS-APNO, G2, and G3 Thermochemical Predictions with Experiment for Formation of Ionic Clusters of Hydronium and Hydroxide Ions Complexed with Water, J. Chem. Phys., 2005, 122 (2), 024302.

95. E.R. Johnson and A.D. Becke, A Post-Hartree-Fock Model of Intermolecular Interactions, J. Chem. Phys., 2005, 123 (2), 024101 .

96. J. Cerny and P. Hobza, The X3LYP Extended Density Functional Accurately Describes H-bonding But Fails Completely for Stacking, PCCP Phys. Chem. Chem. Phys., 2005, 7 (8), $1624-1626$.

97. G.C. Shields, C.A. Laughton, and M. Orozco, Molecular Dynamics Simulations of the d(T.A.T) Triple Helix, J. Am. Chem. Soc., 1997, 119 (32), 7463-7469.

98. G.C. Shields, C.A. Laughton, and M. Orozco, Molecular Dynamics Simulation of a PNA.DNA.PNA Triple Helix in Aqueous Solution, J. Am. Chem. Soc., 1998, 120 (24), 5895-5904.

99. P. Hobza and J. Sponer, Structure, Energetics, and Dynamics of the Nucleic Acid Base Pairs: Nonempirical Ab initio Calculations, Chem. Rev., 1999, 99 (11), 3247-3276.

100. T.N. Lively, M.W. Jurema, and G.C. Shields, Hydrogen Bonding of Nucleotide Base Pairs: Application of the PM3 Method, Int. J. Quantum Chem., 1994, 21, 95-107.

101. J. Sponer, P. Jurecka, I. Marchan, F.J. Luque, M. Orozco, and P. Hobza, Nature of Base Stacking: Reference Quantumchemical Stacking Energies in Ten Unique B-DNA Base-pair Steps, Chem.-A Eur. J., 2006, 12 (10), 2854-2865. 